\title{
Cambio de estado en oraciones de modificación en awajún desde la Teoría de Modelos montagueana
}

\section{Change of State in Awajun Modification Sentences from Montaguean Model Theory}

\author{
Joaquín Antonio Bullón Cornejo \\ Universidad Nacional Mayor de San Marcos, \\ Lima, Perú \\ joaquin.bullon@unmsm.edu.pe \\ orCID https://orcid.org/oooo-0003-0056-7325
}

\author{
Rodrigo Saniel Galloso Cossios \\ Universidad Nacional Mayor de San Marcos, \\ Lima, Perú \\ rodrigo.galloso@unmsm.edu.pe \\ orCID https://orcid.org/oooo-0003-1083-751X
}

\section{Resumen}

El presente trabajo descriptivo-explicativo tiene como objetivo analizar algunas oraciones de modificación de dotación y privación en awajún empleando la Teoría de Modelos montagueana. En primera instancia, se definen aspectos básicos de las acciones de modificación. Luego, se describen aspectos influyentes en la formación de este tipo de oraciones. Estos aspectos son los morfemas de high affectedness y acusativo, así como los papeles semánticos de agente y paciente. Después de esto, se formalizan y analizan algunas oraciones del corpus a través de la propuesta de Richard Montague (1970). Por último, llegamos a la conclusión de que la propuesta de Montague es perfectamente aplicable al awajún.

Palabras clave: lengua awajún; teoría de modelos montagueana; acciones de modificación.

\begin{abstract}
This descriptive-explicative paper has the objective to analyze some endowment and modification sentences in Awajun through the Montaguean Model Theory. In the first instance, basic aspects of modification actions are defined. Then, aspects, influential in the formation of this type of sentences, are described. These aspects are the high affectedness and accusative morphemes, as well as the semantic papers of agent and patient. After that, some sentences of the corpus are formalized and analyzed through Richard Montague's 1970 proposal. Finally, we reach the conclusion that Montague's proposal is perfectly applicable to awajún.
\end{abstract}

Keywords: awajún language; formal semantics; Montaguean Model Theory; modification actions.

\section{Resumo}

O objetivo deste trabalho descritivo-explicativo é analisar algumas sentenças de modificação de dotações e privações em Awajún usando a Teoria do Modelo Montagna. Primeiro, os aspectos básicos das ações de modificação são definidos. Em seguida, são descritos aspectos influentes na formação deste tipo de sentenças. Estes aspectos são os morfemas de alta afetividade e acusação, 
assim como os papéis semânticos de agente e paciente. Depois disso, algumas sentenças do corpus são formalizadas e analisadas através da proposta de Richard Montague (1970). Finalmente, concluímos que a proposta de Montague é perfeitamente aplicável a Awajún.

Palavras-chave: Linguagem de Awajún; teoria do modelo montagueano; ações de modificação.

\section{Introducción}

La lengua awajún es una de las 49 lenguas originarias habladas en el Perú (incluyendo la LSP) y la segunda con mayor cantidad de hablantes en la región amazónica del país, después del asháninka. Esta lengua pertenece a la familia lingüística jíbaro junto a otras lenguas como el wampis, shuar y el achuar-shiwiar. Según el Censo Nacional del INEI (2018), el número total de hablantes de awajún asciende a 56,584. Sobre la distribución espacial de esta lengua, el MINEDU (2013) y el Viceministerio de Interculturalidad (2015) establecen que los hablantes awajún se encuentran en los departamentos de Amazonas, Loreto, Ucayali, San Martín, Cajamarca y Lima (v. gráfico 1). La mayoría de los hablantes se encuentran en el departamento de Amazonas, específicamente en la provincia de Condorcanqui. En la ciudad de Lima, existen comunidades awajún en el distrito de Ventanilla (Solís, 2017).

Gráfico 1. Ubicación del pueblo awajún (Viceministerio de Interculturalidad, 2015)

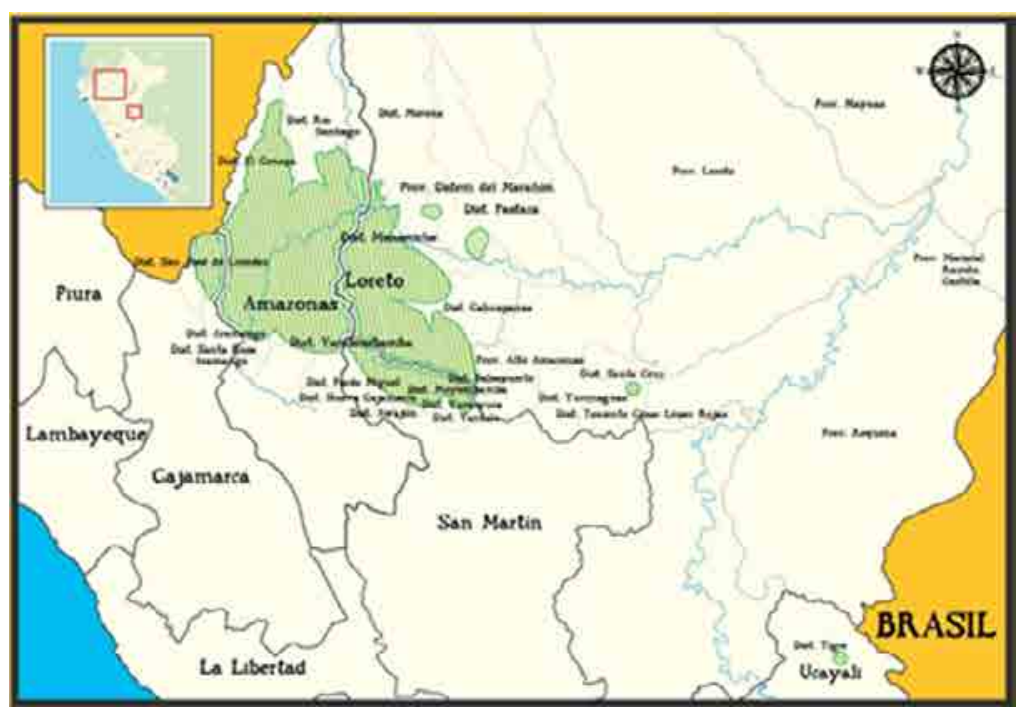


La lengua awajún se caracteriza por ser aglutinante, principalmente sufijante y por seguir la estructura oracional sujeto-objeto-verbo o sov. Simon Overall (2007) la describe como estrictamente nominativa-acusativa, así como con un inventario consonántico de doce fonemas, además de cuatro vocales con nasalidad contrastiva.

Las investigaciones sobre semántica en lenguas indígenas peruanas son pocas y con respecto al awajún, aún más. Sin embargo, tuvimos acceso a dos tesis relacionadas a la lengua y al presente estudio. En primer lugar, tesis de Paz, Polisemia en verbos de percepción visual, auditiva y olfativa del idioma awajún según el enfoque de la semántica cognitiva. (Paz Villavicencio, 2018). Esta tesis analiza algunos verbos perceptivos de la lengua awajún a través de conceptos de la semántica cognitiva, como la prototipicidad y mecanismos metafóricos.

Por otro lado, podemos destacar la tesis de Perez, Análisis morfológico-semántico de los verbos de desplazamiento en taushiro, una lengua en extinción (2008), en la cual se hizo un registro de morfemas verbales de desplazamiento y sus implicancias semánticas en taushiro. Mencionamos esta tesis por el acercamiento que tiene con el presente estudio, pues en los dos se presenta un estudio morfosemántico de las acciones, las cuales se explican más adelante.

La semántica formal se relaciona con el principio de composicionalidad de Frege. Este principio plantea que «el significado de una oración compuesta es una función de los significados de las expresiones componentes» (Lyons, 1997, p. 233), en la cual una función hace referencia una regla matemática que asigna un valor a cada componente, en este caso los lexemas de las oraciones. En otras palabras, cada pieza léxica de una oración aporta un valor de significación para la significación total de la oración. La importancia de este principio radica en que debe existir un principio que explique que el significado total de una oración proviene del significado de cada una de las piezas léxicas, ya que los hablantes no pueden aprender el significado de cada composición léxica (Lyons, 1997).

La teoría de modelos montagueana es un enfoque de la semántica formal que estudia los conceptos de verdad a través de fórmulas lógicas. En esta teoría, se opera con nociones de verdad relativa, es decir la verdad en un modelo, por esto mismo la gramática de Montague está relacionada con la teoría de mundos posibles (Lyons, 1997, p. 250). Es importante mencionar que A. Tarski, en 1933, propuso la teoría de verdad, la cual manifiesta que «una oración $S$ de la lengua L es verdadera sí y solo sí $\mathrm{P}$ » (Moreno Cabrera, 1985, p. 12). Moreno Cabrera menciona que Tarski consideraba que su teoría no era aplicable para las lenguas naturales, 
sin embargo, este fue quien abrió paso a este enfoque. Años más tarde, el lógico estadounidense Richard Montague, en su Gramática Universal (1970), utilizó la teoría de la verdad de Tarski aplicada en lenguas naturales. Cabe mencionar que Montague no diferencia entre las lenguas artificiales y naturales, en el punto que es posible abarcar la sintaxis y semántica de ambas clases de lenguas en una sola teoría (Moreno Cabrera, 1985).

Moreno Cabrera (2003) nos ofrece una descripción y análisis semántico de oraciones a través de la semántica de modelos montagueana. En este libro se explica que los sucesos son resultados de operaciones de caracterizaciones de entidades. Se presenta una clasificación de los sucesos, los cuales se dividen en acciones, procesos y estados. El autor acota que estos tipos de sucesos se encuentran en cualquier lengua y que «la estructura semántica de cualquier oración de cualquier lengua se puede expresar mediante ella».

Al mismo tiempo se explica que los tres tipos de sucesos tienen una relación jerárquica en la que «los procesos se pueden definir a través de estados y acciones, a través de procesos» (Moreno Cabrera, 1985, p. 35). Esta clasificación también se presenta en Chafe (1970), con la diferencia de que este último explica que cada tipo de oración presenta un verbo que determina su tipo de suceso. Esta idea no es recogida por Moreno para el análisis de las oraciones, puesto que un verbo puede expresar los tres tipos de sucesos.

Se infiere que este modelo teórico aplica para todas las lenguas naturales. Esto nos lleva a la siguiente pregunta: ¿se puede aplicar la Teoría de Modelos montagueana en la descripción de oraciones que expresan modificación en awajún? El objetivo principal del presente estudio es analizar las oraciones de modificación del awajún a través de la teoría de modelos montagueana. Para llegar a este objetivo es necesario describir los morfemas que influyen en la formación de oraciones de modificación del awajún, es decir, el morfema de high affectedness $\{-\mathrm{a}(\mathrm{w})\}$ y el de caso acusativo \{-na\}. Por otro lado, también es necesario identificar los papeles temáticos participantes en las oraciones del corpus presentado; así como realizar análisis arbóreos de las oraciones.

204 Lengua y Sociedad. Revista de Lingüística Teórica y Aplicada 


\section{Marco conceptual, referencial o teórico}

\subsection{Acciones de modificación}

Dentro de la clasificación que hace Moreno Cabrera sobre los sucesos, se encuentran las acciones. Moreno Cabrera define a las acciones como relaciones entre procesos y entidades, que se formalizan de la siguiente manera:

a) $\kappa\left(\Upsilon_{2},\left[\wp\left[\Sigma\left(\Upsilon_{1}, \Lambda\right), \Sigma\left(\Upsilon_{1}, \Lambda\right)\right]\right]\right)=$

b) $\aleph\left(\Upsilon_{2},\left[\Sigma\left(\Upsilon_{1}, \Lambda\right) \Rightarrow \Sigma\left(\Upsilon_{1}, \Lambda\right)\right]\right)$

Moreno Cabrera (2003)

Las acciones se clasifican en locomociones, que relacionan entidades con procesos de desplazamiento; y en acciones de modificación, las cuales comprenden las relaciones entre entidades y procesos de mutación. Las modificaciones se subdividen en seis clases, las cuales también se relacionen con un tipo determinado de proceso de mutación. En este estudio, solo formalizamos dos tipos de modificaciones: las dotaciones y las detracciones. Las dotaciones y las privaciones se caracterizan por la atribución y el despojo de características a una entidad por parte de otra. Esto último se puede explicar mejor con los siguientes ejemplos en español:

(1) a. Pedro cocinó el arroz. (El arroz pasó a estar cocinado).

b. Luis desatoró la tubería. (La tubería dejó de estar atorada).

En 1.a podemos encontrar una modificación de dotación puesto que una entidad, el agente Pedro, atribuye la característica de [cocinado] a otra entidad, el objeto arroz. Por otro lado, en 1.b el agente Luis le sustrae la característica de [atorado] a la entidad tubería.

\subsection{Papeles semánticos}

Los papeles semánticos son herramientas utilizadas en diversas teorías lingüísticas que sirven como indicador de las funciones semánticas de los constituyentes de las oraciones. Los papeles semánticos se clasifican de modo distinto según el tipo de suceso de la oración. Dentro de las acciones de modificación podemos encontrar al agente, el cual es el causante de un evento voluntario, y al paciente, el cual se ve afectado por la acción. 


\subsection{Morfema de high affectedness aksionsart -a(w)}

En awajún, el morfema de high affectedness es un morfema verbal que acentúa la «afectación» del objeto (paciente) cuando es usado en verbos transitivos (Overall, 2007, p. 296). Según lo que pudimos notar, este morfema contribuye en la formación de las oraciones de modificación puesto que, cuando es usado en verbos transitivos, el paciente cambia de estado. Existe una diferenciación entre el morfema de high y low affectedness, $\{-$-ini) $\}$, aunque los dos coinciden en el orden morfológico dentro de la palabra. Presentaremos el mismo ejemplo que presentan Overall y Peña, en sus gramáticas de awajún y wampis, respectivamente, para explicar esta diferencia.

(2) pelota-na pelota-acc Pelota exploté

Exploté la pelota (cambio de estado de la pelota).

(3) Hiín hii-na fuego-acc Yo soplé fuego.

Yo soplé el fuego (para mantenerlo vivo, no hay cambio de estado). umpuahai

umpu-a-ha-i

explotar- hiaf-1sg-decl

Este ejemplo también es utilizado por Peña para diferenciar estos dos tipos de morfemas en wampis, lengua de la familia jívaro (Peña, 2015). Como se puede ver en el ejemplo (1), el morfema de high affectedness aparece y existe un cambio de estado de parte del paciente. Por otro lado, el ejemplo (3) presenta el morfema de low affectedness y no existe un cambio de estado por parte del sujeto. Tanto en awajún como en wampis, el morfema de low affectedness puede influir en la formación de oraciones de modificación de mantenimiento en las cuales la entidad mantiene sus propiedades o su estado.

Según la Gramática de Overall, en awajún, verbos típicos como yu «comer» y mau «matar» utilizan el morfema de high affectedness (Overall, 2007, p. 296). Este morfema puede aparecer como $\{-$ aw $\}$ si el morfema siguiente empieza por la vocal -a. Asimismo, el morfema de high affectedness puede tener la forma $\{$-wa $\}$ si es que se le antepone una vocal. Por otro lado, el morfema de low affectedness puede 
aparecer como $\{-\mathrm{i}\}$ o $\{$-ini $\}$ dependiendo del morfema que le sigue. La forma larga aparece cuando precede a los morfemas de pasado inmediato $\{-\nabla\}$ y de pasado distante $\{$-ama...ia\}.

\subsection{Morfema de caso acusativo -n(a)}

Como se mencionó antes, el awajún es una lengua nominativo-acusativa, que utiliza sufijos para marcar los diversos casos de la lengua. Si bien los casos acusativo y nominativo son los más predominantes en la lengua, entre estos dos, el acusativo es el único que presenta una marca con forma fonética. En este caso, se utiliza el morfema $\{-\mathrm{n}(\mathrm{a})\}$ para marcar el caso acusativo y va junto a las bases nominales (Corbera Mori, 1994). Algo curioso del caso acusativo del awajún es que no se marca cuando el sujeto de la oración esté en primera persona plural o segunda persona y que no afecte a un objeto que no esté en primera persona singular (Overall, 2007), como en el siguiente ejemplo extraído de Corbera Mori.
(4) jutíik
néje
yumáitsuji
jutíi-k
néje
yu-mái-tsu-ji
1PL-FOC carne
comer-POT-NEG-1PL

Nosotros carne no comemos.

Nosotros no podemos comer carne.

La lengua awajún puede presentar elisión vocálica en algunas sílabas y esta elisión se puede clasificar en apócope, síncopa y reducción del diptongo (que parece ser una monoptongación sin compensación de tiempo). En este caso, el morfema presentado sufre una elisión por apócope en la cual se elide el núcleo de la última sílaba de la palabra. Esta elisión se puede explicar por el peso silábico, puesto que solo se eliden las vocales de las sílabas ligeras Cv. Es importante mencionar que en awajún la apócope no se presenta en las dos primeras vocales de una palabra, puesto que las palabras mínimas poseen dos vocales.
(5) [datimán
$i k^{\prime} \dot{i n]}$
datima-na
ikinã
ayahuasca-acu
preparar:pfv:seq+3:ss
Ayahuasca preparada.
Habiendo preparado ayahuasca 
Como se ve en el ejemplo (5) tomado de Overall, la marca de acusativo sufre un proceso fonológico de elisión de vocal, con esto la marca es [-n].

\section{Metodología}

El corpus fue obtenido mediante una entrevista y revisión bibliográfica. En total analizamos diez oraciones. Las primeras 8 fueron obtenidas gracias a la amabilidad de nuestro colaborador awajún de iniciales ma a quien pudimos entrevistar por vía telefónica.

MA es un hablante nativo de awajún, docente de Educación Intercultural Bilingüe, por lo que su nivel de fluidez tanto en esta lengua como en el español es tal que permite su uso de forma profesional. Es importante mencionar que las oraciones obtenidas en este recojo de datos de parte de nuestro colaborador siguen la estructura svo frente a la esperable forma sov de la lengua, siendo posible una influencia de su bilingüismo en estos datos.

Las oraciones restantes fueron obtenidas de la tesis A grammar of Aguaruna de Simon Overall (2007) y del Diccionario Awajún-Castellano de los compiladores Nexar Asangkay Sejekam y Edwardo Gomez Antuash (2008).

En lo que respecta a las limitaciones que tuvo este trabajo en su realización, destaca la influencia que la vigente situación de pandemia causada por el virus SARS-CoV-2 ha tenido, pues las medidas de seguridad requeridas en la prevención de su contagio restringieron el número de colaboradores posibles. Asimismo, por las ya mencionadas medidas, así como que el colaborador careciera de conexión a internet en su actual ubicación, tampoco se pudo realizar una entrevista física, por lo que la comunicación se realizó únicamente por vía telefónica. Este último hecho, a causa de la deplorable señal y audio obtenido, fue a su vez desfavorable en la transcripción de dichos diálogos para el corpus de este trabajo. Por todo esto mencionado, agradecemos profundamente a nuestro colaborador awajún, quien tuvo una gran paciencia para brindarnos los datos a pesar de las circunstancias.

\section{Análisis}

La presente sección está organizada en el correspondiente análisis de tres oraciones. Primero, se hace un análisis morfológico de las misma. Luego se muestra un diagrama arbóreo y, por último, la formalización de la oración. En la última subsección, se encuentran los resultados.

208 Lengua y Sociedad. Revista de Lingüística Teórica y Aplicada 


\subsection{Atash yuwae bagan}

(6) Atash yuwae atash $\quad \mathrm{yu}-\mathrm{a}-\mathrm{i}$

bagan.

gallina comer-hiaf-decl

baga-na

"Gallina comió gusano»

gusano-acu

«La gallina comió al gusano»

Como se puede ver en (6), el análisis morfológico de la oración, el sufijo de high affectedness, $-\mathrm{a}(\mathrm{w})$, aparece como $\{-\mathrm{wa}\}$ puesto que la raíz del verbo yu («comer») termina en vocal. Por otro lado, el sufijo de caso acusativo $\{$-na $\}$ aparece como $\{-n\}$, puesto que sufre el proceso de elisión vocálica.

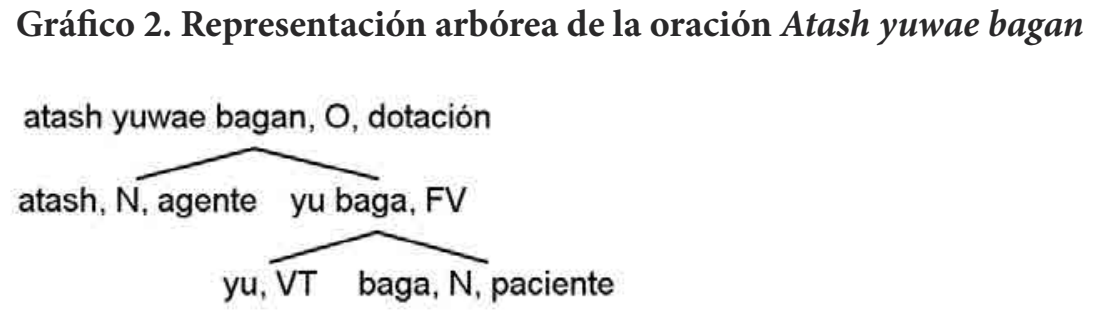

Gráfico 2. Representación arbórea de la oración Atash yuwae bagan atash yuwae bagan, $\mathrm{O}$, dotación

El diagra ma arbóreo refleja que el argumento que se relaciona más con el verbo es el paciente, esto nos puede decir que el proceso El gusano fue comido (por el agente) es subyacente a la acción de la oración.

En esta oración, la acción origina el proceso denotado por El gusano fue comido (por el agente). La formalización de esta oración de modificación de dotación es la siguiente:

$\aleph\left(\right.$ atash, $\left[\left(\neg \sum\left((\right.\right.\right.$ baga, yuama $) \Rightarrow \sum$ (baga, yuama) $\left.\left.)\right]\right)$

Donde $\aleph$ indica una relación de acción, $\Sigma$ un estado $\mathrm{y} \Rightarrow$ un proceso. Entonces, la acción de comer se explica a partir de un proceso que afecta al paciente $y$, a su vez, este proceso se explica por el cambio de estado denotado por el verbo. 


\subsection{Mina apang mae kamitan}

$\begin{array}{llll}\text { (7) } & \text { Mina } \quad \text { apang } & \text { mae } & \text { kamitan } \\ \text { mi-na } & \text { apang } & \text { ma-i } & \text { kamita-na } \\ \text { 1sg-acu padre } & \text { matar+hiaf-decl } & \text { gamitama-acu } \\ \text { «Mi padre mató gamitama» } & \\ \text { «Mi padre pescó el gamitama» } & \end{array}$

El análisis de (7) nos muestra al sufijo de high affectedness junto al verbo la raíz del verbo mau («matar»). Por otro lado, el sufijo de caso acusativo aparece en su forma $\{-\mathbf{n}\}$ por la elisión vocálica. En este caso, la oración presenta el verbo mau «matar» en uno de sus significados en awajún. En este caso, el verbo hace referencia a pescar. Podemos decir que la polisemia de este verbo reside en el significado de «quitar la vida», entonces en la formalización se evidencia el paso de «vivo» o iwaaku en awajún a «muerto» o jakáu.

\section{Gráfico 3. Representación arbórea de la oración Mina apang mae kamitan}

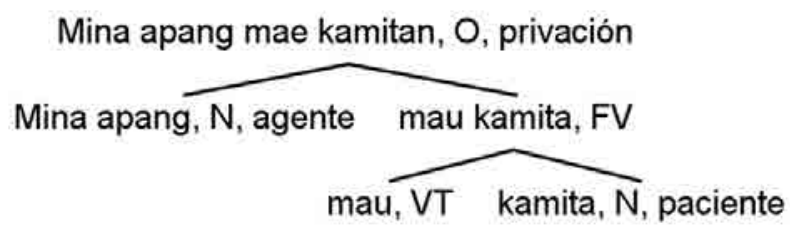

Esta oración presenta una modificación de privación, puesto que el proceso que origina la acción es El gamitama fue matado (por el agente). El diagrama arbóreo presentado refleja que la $\mathrm{FV}$ es subyacente de la oración. La formalización de esta oración de privación es la siguiente:

$$
\aleph(\text { wii, }[(\Sigma((\text { kamita, iwaaku }) \Rightarrow \Sigma(\text { kamita, jakáu) })])
$$




\subsection{Ikam yawa mae Dikain}
(8) Ikam yawa
mae
Dikain
ikama yawa
ma-i
Miguel-na
selva perro
matar+hiaf-decl
Miguel-acu
«El perro de la selva mató Miguel».
«El jaguar mató a Miguel».

En (8), la raíz del verbo matar se encuentra fusionada con el sufijo de high affectedness, al igual que en los demás casos el sufijo de caso acusativo pierde la última vocal y queda la forma $-n$.

Al igual que en los otros casos, el diagrama arbóreo muestra que el proceso Dikei fue matado (por el agente) es subyacente a la oración.

\section{Gráfico 4. Representación arbórea de la oración Ikam yawa mae Dikain}

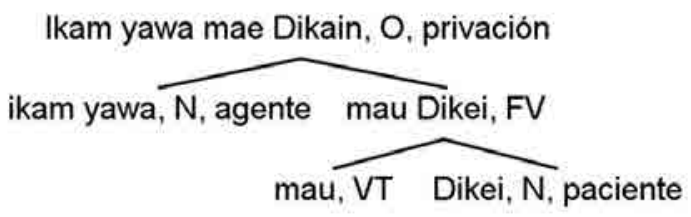

Esta oración presenta una modificación de privación puesto que el proceso que origina la acción es Dikei fue matado (por el agente). Al igual que en (7), la formalización muestra el paso del objeto de «vivo» o iwaaku a «muerto» o jakáu en awajún. La formalización de esta oración de modificación de privación es la siguiente:

א (Ikam yawa, $[(($ Dikain, iwakku) $\Rightarrow \Sigma($ Dikei, jakáu $))])$ 


\title{
4.4 Resultados
}

\author{
Atash yuwae bagan \\ Mina apang mae kamitan \\ Ikam yawa mae Dikain
}

La cita de Overall "The high affectedness suffix accentuates the affectedness of the object when used with transitive verbs» (Overall, 2007) da a entender que se producirá el cambio de estado del paciente cuando el sufijo de high affectedness se encuentre con un verbo transitivo.

Este cuadro refleja que el verbo y el paciente de las oraciones de modificación de dotación y privación del awajún poseen las marcas de high affectedness y del caso acusativo, respectivamente. Esto último confirma la cita de Overall, puesto que el paciente de la oración llega a cambiar de estado cuando los sufijos antes mencionados aparecen.

\section{Conclusiones}

Después de haber descrito los morfemas que influyen en la formación de oraciones de modificación de dotación y privación, morfema de high affectedness y del caso acusativo; haber identificado a los papeles semánticos participantes en este tipo de oraciones, papel agente y paciente; y de realizar los árboles sintácticos que relacionan la sintaxis y la semántica podemos resolver el objetivo general del trabajo.

La principal conclusión a la que se puede llegar es que la teoría de modelos montagueana es perfectamente aplicable en la lengua awajún, habiéndosela usado de forma efectiva en el presente trabajo para explicar los cambios de estado en las oraciones de modificación estudiadas.

La aplicación de la teoría de modelos montagueana, y de la semántica formal en general, da luces a posibles aplicaciones dentro de la lingüística computacional. El artículo aquí presentado muestra la relación entre un lenguaje formal y una lengua natural, el awajún. De este modo, la aplicación de la lingüística computacional es posible no solo en esta lengua, sino en más lenguas amazónicas y originarias. 


\section{Agradecimientos}

Los autores agradecen los alcances brindados por la lingüista Verónica Lazo García, profesora del curso Semántica II en la Universidad Nacional Mayor de San Marcos. También es oportuno señalar un agradecimiento a nuestro colaborador de iniciales ma por la ayuda y paciencia brindada.

\section{Financiamiento}

La investigación se realizó sin financiamiento.

\section{Conflicto de intereses}

Los autores no presentan conflicto de interés.

\section{Referencias bibliográficas}

Asangkay Sejekam, N., \& Gomez Antuash, E. (2008). Awajún chícham jugbau. Diccionario Awajún-Castellano (parcial) (Versión Preliminar ed.). Amazonas, Perú.

Chafe, W. L. (1970). Meaning and the Structure of Language. Chicago, Estados Unidos: The University of Chicago Press.

Corbera Mori, A. (1998). Características Morfosintácticas del Aguaruna (Jíbaro). Campinas, Brasil: Universidade Estadual de Campinas.

Corbera Mori, A. H. (1994). Fonologia e Gramática do Aguaruna (Jívaro). Campinas, Brasil: Universidade Estadual de Campinas (UnicAmP).

Instituto Nacional de Estadística e Informática-INEI. (2018). Perfil Sociodemográfico del Perú, elaborado a partir de los Censos Nacionales XII de Población, VII de Vivienda y III de Comunidades Indígenas. Lima, Perú. Obtenido de https:// www.inei.gob.pe/media/MenuRecursivo/publicaciones_digitales/Est/Lib1539/ libro.pdf.

Lyons, J. (1997). Semántica lingüística: una introducción. Barcelona, España: Ediciones Paidós.

Menegotto, A. (s.f.). Léxico, sintaxis y semántica. Roles semánticos.

Ministerio de Educación del Perú. (2013). Documento Nacional de Lenguas Originarias del Perú (Primera Edición ed.). Lima, Perú. Obtenido de http:// www2.minedu.gob.pe/filesogecop/DNLversion\%2ofinal\%2owEB.pdf

Moreno Cabrera, J. C. (1985). Lógica formal y lingüística. Una introducción a la gramática de R. Montague. Madrid, España: Ediciones de la Universidad Autónoma de Madrid. 
Moreno Cabrera, J. C. (2003). Semántica y Gramática. Sucesos, papeles sintácticos y relaciones sintácticas. Madrid, España: A. Machado Libros.

Overall, S. (2007). A Grammar of Aguaruna. Sydney, Australia: Research Centre for Linguistic Typology.

Payne, D. (1978). Nasalidad en aguaruna. Pucallpa: Instituto Lingüístico de Verano.

Paz Villavicencio, J. (2018). Polisemia en verbos de percepción visual, auditiva y olfativa del idioma awajún según el enfoque de la semántica cognitiva. Lima, Perú: Universidad Nacional Mayor de San Marcos.

Peña, J. (2015). A grammar of wampis. University of Oregon. Obtenido de http:// hdl.handle.net/1794/19730

Perez, J. (2008). Análisis morfológico-semántico de los verbos de desplazamineto en taushiro. Lima, Perú: Universidad Nacional Mayor de San Marcos.

Quesada, D. (1989) Lingüística Computacional y Semántica Formal. Theoria: An International Journal for Theory, History and Foundations of Science, 4, pp. 269-274.

Solís, G. (2017). Pueblos indígenas en Lima: la ciudad como maloca. Lengua y Sociedad. 13(1), 55-69. Obtenido de http://revista.letras.unmsm.edu.pe/index. $\mathrm{php} / \mathrm{ls} /$ article/view/438.

Viceministerio de Interculturalidad. (2015). Mapa $\mathrm{N}^{\circ}$ 2. Pueblo awajún. En MinCultura, Los pueblos achuar, awajún, kandozi y wampis (pág. 16). Lima, Perú: LucentPerú SAC.

Wipio Deicat, G., Paati Antunce, A., \& Jakway, M. (1996). Diccionario AguarunaCastellano. Lima, Perú: Instituto Lingüístico de Verano.

$\begin{array}{ll}\text { Abreviaturas utilizadas } \\ \text { acu } & \text { acusativo } \\ \text { decl } & \text { declarativo } \\ \text { ss } & \text { mismo sujeto } \\ \text { hiaf } & \text { high affectedness Aktionsart } \\ \text { loaf } & \text { low affectedness Aktionsart } \\ \text { pfv } & \text { perfectivo } \\ \text { seq } & \text { secuencial }\end{array}$

214 Lengua y Sociedad. Revista de Lingüistica Teórica y Aplicada 
CambiodeestadoenoracionesdemodificaciónenawajúndesdelaTeoríadeModelosmontagueana Joaquín Antonio Bullón Cornejo y Rodrigo Saniel Galloso Cossios

\section{Trayectoria académica de los autores}

\section{Joaquín Bullón}

Actualmente es estudiante de la carrera de Lingüística en la Universidad Nacional Mayor de San Marcos. Tiene interés en el estudio lingüístico de las lenguas de señas del país, del awajún y en la Fonología en general.

\section{Rodrigo Galloso}

Actualmente, es estudiante de la carrera de Lingüística en la Universidad Nacional Mayor de San Marcos. Tiene interés en el estudio lingüístico de las lenguas de señas del país, del awajún y en la Fonología en general. 